\title{
Comparison of Three Screening Methods for Cervical Cancer in China - A Systematic Study of 3,300 Cases in Rural Areas of Shanxi Province
}

\section{Dong Yan ${ }^{1 *}$, Bai Jigeng ${ }^{1 *}$, Zhang Yuping ${ }^{1}$, Shang Guang Jie ${ }^{2}$, Zhao Yan ${ }^{1}$ and Dongfeng Tan ${ }^{3}$}

${ }^{1}$ Shanxi Maternal and Child Health Care Hospital, Shanxi, Taiyuan, PR China

${ }^{2}$ Beijing Fulin Guangzhi Medical Technology Co Ltd, Beijing, PR China

${ }^{3}$ UT MD Anderson Cancer Center, Huston, Texas, USA

\begin{abstract}
Objective: Reaching women in rural China is one of the major public health problem today and even though various methodologies were introduced they had limited or no success. The goal of our study was to investigate the usefulness of DNA quantitative assessment (ploidy) in comparison with conventional cytology (LBC) and HPV testing and its possible role in the clinical practice for rural women.

Methods: In collaboration with Shanxi Women and Children Health Center we finished a study in Shanxi Province (3300 women) using Image cytometer. Each women underwent HPV testing, liquid based cytology screening (LBC) using manual and semi-automated analysis based on DNA ploidy and EA50 staining (on the same slide). All positive cases by any method were invited for colposcopy and biopsy.

Results: Our results showed that DNA ploidy in combination with EA50 have superior performance in comparison with LBC or HPV method.

Conclusion: Automated combined qualitative and quantitative image cytometry is a very good candidate for screening in low resource settings in China where the conventional cytology or LBC reading cannot be performed due to the lack of adequate infrastructure and trained cytotechnologists or cytopathologists. Furthermore digitalization of images open a door for data transfer and cloud computing which in turn can improve access to proper medical care in rural China.
\end{abstract}

Keywords: Cervical cancer screening; DNA ploidy; Automated analysis; Cytology

\section{Background}

Cervical cancer is a high incidence cancer. Particularly, in China, it represents the commonest malignancy among the female reproductive organ systems. The Chinese registry reported an age-adjusted cervical cancer incidence rate of 8.9 per 100,000 women in China with approximately 131,500 new cases every year, and thus accounts for $28.8 \%$ of the overall world's new cases $[1,2]$. Incidence rates in young women are growing approximately at a rate of $2-4 \%$ per year [3]. The prevalence of cervical cancer in China increased from 8.2/100,000 in 1999 to $15.1 / 100,000$ in 2010, and in Shanxi province the rate was $40.7 / 100,000$ in 2010, the highest in the country [4]. The numbers must be taken with caution, because of the high disparity between urban and rural areas therefore the national mortality and incidence rates should be interpreted cautiously [5]. The numbers clearly show that early screening and detection of cervical precancerous lesions together with the early treatment is an important preventative healthcare measure.

From 2011-2014, we performed screening of 1.349 million women aged 35-64, in the rural areas in Shanxi province. According to our own data, cervical cancer detection rate was $6.1 / 10,000(816 / 1,349,000)$ and the precancerous lesion detection rate was $34.62 / 10,000$ $(4,669 / 1,349,000)$. The detection of pre-cancerous lesions was relatively lower compared to publish data, indicating that primary healthcare institutions need to do more to improve their approach to cervical cancer screening.

Finding a safe, reliable, easy, and affordable screening method to screen cervical cancer for a large-scale population became our top priority. In our study, we compared three methods suggested previously for screening [6,7]. Conventional liquid-based cytology (LBC), HPV detection, and automated combined DNA ploidy using image analysis.
We compared test results against colposcopy and biopsy diagnoses, which ranged from cervical intraepithelial neoplasia (CIN) to cancer. We evaluated each test application and cost/benefit value for a largescale cervical cancer screening programs. We also looked at the ASCUS group and how it compared when detection is done by each of three methods.

\section{Materials and Methods}

\section{General information}

From October to December 2014, we randomly selected 3,300 rural women at the age range from 35 to 64 from four counties in Shanxi province, who were also participating in the HPV pilot project. These women were screened for cervical cancer by three screen modalities, namely LBC, HPV-care, and combined DNA ploidy with EA50 stain analysis. If a sample was positive with any of the three test methods,

*Corresponding authors: Jigeng B, Maternal and Child Health Hospital, No. 13 Xinmin North Street, Xinghualing District, Taiyuan, Shanxi Province, PR China, Tel: 13994250697; E-mail: zhang_yp510@163.com

Yan D, Maternal and Child Health Hospital, No. 13 Xinmin North Street, Xinghualing District, Taiyuan, Shanxi Province, PR China, Tel: 13753114960; E-mail: ady3360@126.com

Received November 15, 2017; Accepted December 20, 2017; Published December 26, 2017

Citation: Yan D, Jigeng B, Yuping Z, Jie SG, Yan Z, et al. (2017) Comparison of Three Screening Methods for Cervical Cancer in China - A Systematic Study of 3,300 Cases in Rural Areas of Shanxi Province. J Cytol Histol 8: 492. doi: 10.4172/2157-7099.1000492

Copyright: (C) 2017 Yan D, et al. This is an open-access article distributed unde the terms of the Creative Commons Attribution License, which permits unrestricted use, distribution, and reproduction in any medium, provided the original author and source are credited. 
we invited women for colposcopy and subsequent biopsy to obtain a histopathological diagnosis, which is considered gold standard.

\section{Methods}

Collection of samples for conventional cytology and combined DNA ploidy analysis: It was done with collection kits provided by Landing Cervical Cancer Screening Center, Wuhan, China. The collection kits included a cervical brush, a jar with fixative and requisition form with the same bar code as a bar-code on the jar with fixative for easy computer entry and identification, as well as quality assurance. Briefly, the standard procedure is as follows: Using a vaginal dilator to provide a direct visualization of cervix, a specially trained gynecologist collected samples at designated clinics. Cervical brush was rotated clockwise 3-5 times around the cervix to collect epithelial cells and then the head of the brush was detached and placed in the jar with fixative, after which it was shook gently 3-5 times to release the cells from the brush into the fixative solution. After verifying the information on the jar's label and requisition form, the sample was sent to Shanxi Maternal and Child Hospital for further preparation, slide staining and analysis.

Collection of samples for high-risk HPV testing: It was done by using the HPV-care brush from Qiagen Bioengineering Co., Ltd. Germany. The brush was inserted into the cervical canal and rotated 3 times in the counterclockwise direction, according to the manufacturer instructions, placed into a special medium provided by Qiagen Bioengineering Co Ltd, ID verified by the staff, and sent to the designated laboratory for testing.

Liquid-based thin layer preparation and staining: Each cytology sample was used to make two slides, one for conventional cytology and one for automated combined DNA ploidy analysis. The conventional cytology slide was stained with Papanicolaou stain, while the DNA ploidy analysis slide was stained with a DNA-specific staining kit provided by Lanading Cervical Cancer Screening Center Hubei, Wuhan. The kit contained detailed instruction about two step staining process. The first step was DNA specific stain followed by EA50 staining of the same slide.

High-risk HPV testing with HPV-care: was done using Qiagen Bioengineering's second generation American hybrid capture method (HC2). The HPV-DNA mixed probe kit used a 96-well plate to detect 14 different subtypes of $\operatorname{HPV}(16,18,31,33,35,39,45,51,52,56,58$, 59,66 , and 68 ), with $1 \mathrm{pg} / \mathrm{mL}$ of HPV DNA as the criteria for a positive diagnosis.

Conventional cytology: All Papanicolaou-stained slides were independently read by at least 2 cytopathologists from our hospital. The slides were assessed according to the five diagnostic categories of the revised Bethesda System (TBS): 1. Normal or Benign, 2. Atypical Squamous Cells of Unknown Significance (ASCUS), 3. Low Grade Squamous Intraepithelial Lesion (LSIL), 4. High Grade Squamous Intraepithelial Lesion (HSIL), and 5. Squamous Cell Carcinoma (SCC). For quality control purposes, we randomly selected $20 \%$ of positive and $10 \%$ of negative slides and assessed them again.

Automated DNA ploidy analysis with EA50 counterstaining: Slides were stained with a DNA-specific staining kit (Wuhan Landing Cervical Cancer Screening Center, Wuhan, China) using modified Feulgen reaction with Thionin. As a second step the same slides were stained with EA50 in order to make cell cytoplasm visible. After coversliping slides were scanned by an automated image cytometer Cyto System (Wuhan Landing Cervical Cancer Screening Center). The system captured images from 1,080 fields of view, and from each object captured the amount of DNA was measured together with over 400 different nuclear and cell features. The system is designed to recognize nuclei of each cell based on indexed data set in the background. DNA content was expressed as a quantitative DNA index with a unit of C. Cells in the G0 (resting) and G1 (gap 1) phases have 23 pairs of chromosomes in the nucleus and are referred to as diploid, or $2 \mathrm{C}$ cells. In the $\mathrm{S}$ phase (DNA synthesis), there are between 23 and 46 pairs of chromosomes, therefore some nuclei may appear "aneuploidy". For example, 3C cells or 4C (tetraploid) nuclei may be in the G2 (gap 2) phase of late stage DNA synthesis. The diagnostic criteria for abnormal DNA ploidy was $5 \mathrm{C}$ or above, i.e. over 2.5 times the normal amount of DNA. In addition the analytical software in cytometer combines entire cell morphology characteristics as well.

Colposcopy and pathology evaluation: All women with ASCUS or worse lesions in LBC, positive in HPV-care testing, or had cells with DNA ploidy of over 5C in quantitative DNA ploidy analysis were referred to colposcopy and subsequent biopsy of suspicious areas, performed by specially trained and experienced colposcopy doctors. If biopsy was taken that specimens was placed in $10 \%$ formalin fixative, and after verification of the labels and requisition forms to ensure correct ID, sent for examination by a qualified pathologist in the pathology department of Shanxi Medical University. The pathologist issued a diagnostic report, and the chief pathologist of Shanxi Province Cancer Hospital performed quality control checkup on a randomly selected subset of samples.

Pathology (histology) classifications were classified as follows: Mild Cervical Intraepithelial Neoplasia (CIN I), Moderate Cervical Intraepithelial Neoplasia (CIN II), High-grade Cervical Intraepithelial Neoplasia (CIN III), and invasive Cervical Cancer (CC).

\section{Results}

We calculated the detection rate of LBC (ASCUS or worse lesion), HPV-care positive, and abnormal DNA ploidy. LBC positive detection rate was $5.26 \%$, with a diagnosis accuracy rate of $22.49 \sim 54.55 \%$; HPV positive detection rate was $16.85 \%$, with a diagnostic accuracy rate of $63.64 \sim 100 \%$; DNA ploidy positive rate was $4.83 \%$, with a diagnostic accuracy rate of $85.71 \sim 100 \%$. Table 1 summarizes the positive rates detected by three tested methods.

Using all three methods on the 3,300 cases, 88 cases (2.67\%) of CIN I+ were detected. The details are summarized in Table 2 . Of these, 47 cases $(1.42 \%)$ were CIN I and 41 cases $(1.24 \%)$ were CIN II+. There was only 1 case $(0.03 \%)$ of cervical carcinoma.

484 colposcopies were performed for rural women who were found to be positive by either test during the screening, and 251 biopsies were performed. Using the pathological diagnosis results as the gold standard, we calculated the sensitivity, specificity, positive predictive value (PPV), and negative predictive value (NPV) for diagnoses of CIN II and above for each of the three tests. LBC: sensitivity was $18.75 \%$, specificity was $89.16 \%$, PPV was $29.03 \%$, NPV was $82.27 \%$; HPV-care: sensitivity was $85.42 \%$, specificity was $28 \%$, PPV was $22.16 \%$, NPV was $80.65 \%$; DNA ploidy (cell with nuclei of $5 \mathrm{C}$ or above abnormal ploidy): sensitivity was $72.92 \%$, specificity was $70.44 \%$, PPV was $36.84 \%$, NPV was $91.67 \%$. The data are summarized in Table 3.

If we observe results from the 251 cervical biopsies, in the light of only one test applied, with LBC only, the number of missed diagnoses would be 31 cases of CIN I, 21 cases of CIN II, 17 cases of CIN III, and 1 case of cervical carcinoma; with only HPV-care, the would be missed 


\begin{tabular}{|c|c|c|c|c|c|}
\hline Screening method & Number of cases & Number of positive cases & Positive detection rate & Inadequate samples & Inadequate sample rate \\
\hline HPV & 3300 & 556 & $16.85 \%$ & - & _ \\
\hline LBC & 3253 & 171 & $5.267 \%$ & 132 & $4.06 \%$ \\
\hline DNA & 3253 & 152 & $4.83 \%$ & 105 & $3.23 \%$ \\
\hline
\end{tabular}

Table 1: Comparison of positive detection rates between the three methods.

\begin{tabular}{|c|c|c|c|}
\hline Screening method & Number of cases & CIN I+ & Positive detection rate (/100 000) \\
\hline DNA & 3253 & 34 & $166.0 / 10,000$ \\
\hline LBC & 3253 & 66 & $101.4 / 10,000$ \\
\hline HPV & 3300 & 88 & $200.0 / 10,000$ \\
\hline All methods & 3300 & $266.7 / 10,000$ \\
\hline
\end{tabular}

Table 2: Comparison of positive detection rates and CIN I+ data.

\begin{tabular}{|c|c|c|c|c|c|}
\hline Screening method & Screening result & Sensitivity & Specificity & PPV & NPV \\
\hline \multirow[t]{3}{*}{ LBC } & ASCUS & $18.75 \%$ & $89.16 \%$ & $29.03 \%$ & $82.27 \%$ \\
\hline & LSIL & $4.17 \%$ & $98.03 \%$ & $33.33 \%$ & $81.22 \%$ \\
\hline & HSIL & 0 & $99.01 \%$ & 0 & 80.72 \\
\hline \multirow[t]{3}{*}{ DNA } & $<3$ & $72.92 \%$ & $70.44 \%$ & $36.84 \%$ & $91.67 \%$ \\
\hline & 3to9 & $52.08 \%$ & $88.18 \%$ & $51.02 \%$ & $88.61 \%$ \\
\hline & $\geq 10$ & $25.00 \%$ & $98.03 \%$ & $75.00 \%$ & $84.68 \%$ \\
\hline HPV & Positive & $85.42 \%$ & $28 \%$ & $22.16 \%$ & $80.65 \%$ \\
\hline
\end{tabular}

Table 3: Comparison of the three methods in detection of CIN II+ cases (\%).

\begin{tabular}{|c|c|c|c|c|c|c|}
\hline \multirow[t]{2}{*}{ Pathology diagnosis } & \multirow[t]{2}{*}{ Cases } & \multicolumn{4}{|c|}{ LBC } & \multirow[t]{2}{*}{ Positive rate (\%) } \\
\hline & & Inflammation & ASCUS & LSIL & HSIL & \\
\hline Inflammation & 162 & 150 & 8 & 2 & 2 & $12(7.41)$ \\
\hline CIN I & 41 & 31 & 10 & 0 & 0 & $10(24.39)$ \\
\hline CIN II & 26 & 21 & 4 & 1 & 0 & $5(19.23)$ \\
\hline CIN III & 21 & 17 & 3 & 1 & 0 & $4(19.05)$ \\
\hline Carcinoma & 1 & 1 & 0 & 0 & 0 & $0(0)$ \\
\hline Total & 251 & 220 & 25 & 4 & 2 & $31(12.35)$ \\
\hline
\end{tabular}

Table 4: Comparison of conventional cytology with pathology diagnoses.

diagnoses were 9 cases of CIN I, 3 cases of CIN II, and 4 cases of CIN III; with DNA ploidy, the number of would be missed diagnoses was 17 cases of CIN I, 11 cases of CIN II, and 2 cases of CIN III. 3. Tables 4-6 list the detailed results of the comparisons.

In our study the HPV infection rate was $16.85 \%$. Due to the high sensitivity of HPV detection tests, we found $556 \mathrm{HPV}$ positive cases. Since every positive case would need to be followed by cytology testing, and subsequently colposcopy, and biopsy, this would result in an increased workload for ob/gyn specialist and most likely high economic burden with a low economic efficiency. Coupled with primary physicians' limited knowledge in this area, the high number of false positives would also cause undue stress to the patients. We also looked how combined tests performed for pathology diagnosis CIN I and worse lesions. The data are summarized in Table 7.

In our study, $4.06 \%$ of LBC slides were inadequate (unsatisfactory), compared with only $3.23 \%$ DNA ploidy slides that were inadequate. In our opinion, during a large-scale screening project with huge numbers of participants, the number of inadequate samples can be a matter of concern. Please refer to Table 1.

Next, to identify the most economically efficient screening method, we looked at the operating cost of each test and calculated the costbenefit ratios of each different method. For this large-scale program, the fees were as follows: $\mathrm{LBC}$ was $30 \mathrm{RMB} /$ person, DNA ploidy analysis using image cytometry was $30 \mathrm{RMB} /$ person, and HPV-care was 70 $\mathrm{RMB} /$ person. We also calculated the cost of detecting each case of CIN. We found that the average cost was 3595.25 RMB for LBC, 4510.70
RMB for HPV-care, and 2156.02 RMB for DNA ploidy. The cost efficient evaluation of three methods is summarized in Table 8 .

Over the last a few decades several methods have emerged for cervical cancer screening: Pap smear, Liquid based cytology (LBC), HPV testing and DNA ploidy by image or flow cytometry.

Pap smear, although still being used worldwide with various successes, traditionally has a high false negative rate (approximately15-40\%) [2] due to various factors, such as from specimen collection, smear technique, proper fixation or lack of it, requirement for skilled staff, among others. Subsequently, with the introduction of liquid based cytology and thin preparation of slides as a monolayer, improvement of the quality of sample preparation has achieved. This methodology has allowed cytologist to observe fine cell deposition. This has led to overall improvement of slide examination and eventually the accuracy of diagnosis, compared with the traditional Pap smears. With accepted notion that HPV is the most important risk factor for cervical cancer pathogenesis, a new era of testing has emerged. However, HPV infection requires a long latent period before cervix shows any recognizable precancerous changes. Furthermore, following infection with HPV, most of the infections would resolve without leaving any notable consequences on cervical mucosa cells. These factors, some relevant to the cost efficiency, should be considered when HPV-based screen is to be implemented. Screening of precancerous lesions and cervical cancer with automated image cytometer (cell DNA ploidy) utilizing quantitative analysis system has been reported previously and perhaps it is currently most extensively used across China [8,9]. 


\begin{tabular}{|c|c|c|c|c|c|c|}
\hline \multirow[t]{2}{*}{ Pathology diagnosis } & \multirow[t]{2}{*}{ Cases } & \multicolumn{4}{|c|}{ Number of cells with DNA ploidy of $>5 \mathrm{C}$} & \multirow[t]{2}{*}{ Positive rate (\%) } \\
\hline & & None & $<3$ & 09-Mar & $\geq 10$ & \\
\hline Inflammation & 162 & 126 & 23 & 10 & 3 & $36(22.22)$ \\
\hline CINI & 41 & 17 & 13 & 10 & 1 & $24(58.54)$ \\
\hline CINII & 26 & 11 & 5 & 7 & 3 & $15(57.69)$ \\
\hline CINIII & 21 & 2 & 4 & 6 & 9 & $19(90.48)$ \\
\hline Carcinoma & 1 & 0 & 1 & 0 & 0 & $1(100)$ \\
\hline Total & 251 & 156 & 46 & 33 & 16 & $95(37.85)$ \\
\hline
\end{tabular}

Table 5: Comparison of DNA ploidy with pathology diagnoses.

\begin{tabular}{|c|c|c|c|c|}
\hline \multirow{2}{*}{$\begin{array}{c}\text { Pathology } \\
\text { diagnosis }\end{array}$} & \multirow{2}{*}{ Cases } & \multicolumn{2}{|c|}{ HPV testing } & \multirow{2}{*}{ Positive rate (\%) } \\
\cline { 3 - 5 } & & Negative & Positive & \\
\hline Inflammation & 162 & 50 & 112 & $112(69.14)$ \\
\hline CINI & 41 & 9 & 32 & $32(78.05)$ \\
\hline CINII & 26 & 3 & 23 & $23(88.46)$ \\
\hline CINIII & 21 & 4 & 17 & $17(80.95)$ \\
\hline Carcinoma & 1 & 0 & 1 & $1(100)$ \\
\hline Total & 251 & 66 & 185 & $185(73.71)$ \\
\hline
\end{tabular}

Table 6: Comparison of HPV with pathology diagnoses.

\begin{tabular}{|l|c|c|c|}
\hline $\begin{array}{l}\text { Pathology } \\
\text { diagnosis }\end{array}$ & $\begin{array}{c}\text { LBC(-) } \\
\text { DNA (+) and HPV } \\
(+)\end{array}$ & $\begin{array}{c}\text { DNA (-) } \\
\text { TCT (+) and HPV } \\
(+)\end{array}$ & TCT (+) and DNA (+) \\
\hline CINI & 12 & 1 & 0 \\
\hline CINII & 12 & 1 & 1 \\
\hline CINIII & 12 & 0 & 1 \\
\hline Carcinoma & 1 & 0 & 0 \\
\hline
\end{tabular}

Note: TCT (-) indicates that the cervical liquid-based cytology is normal or inflammatory, LBC (+) indicates that the cervical liquid-based cytology is ASCUS or above; DNA (-) indicates no DNA aneuploidy cell found, and DNA (+) represents DNA aneuploidy found.

Table 7: LBC(-), DNA (-), and HPV (-) compared with pathology diagnoses.

Since 2009, the State Health Commission (former Ministry of Health) began implementing major public health projects aimed to improve health for women and children in China. By the end of 2014 more than 40 million rural women participated in cervical cancer screening. Since most of the work was done in rural areas where hospitals lacked appropriate equipment, technology, and adequate professionals, it was challenging and very difficult to ensure the quality of such screening. In other words, most of the primary healthcare facilities lack cytopathologists and pathologists. In a few local hospitals, sometime only trained technicians perform the task. For some clinics and small rural hospitals, even if they have pathologists and cytopathologists, those medical professionals usually lack formal training for quality cytology evaluation. Such cervical cancer screening is prone to human error, poor repeatability, and other quality issues.

Due to lack of cytology trained staff, there are substantial limitations in implementing liquid based cytology (LBC) which relays on knowledge about cell morphological and structural features [10]. It was reported that the accuracy of detecting CIN varies from $67 \%$ to $92 \%$, [11] with about $30 \%$ of CINs being missed if only a single cytology exam is performed [11].

In our study, biopsy results were used as a gold standard for the final diagnosis. Unfortunately in our study we did not have a luxury of taking random biopsies of completely negative cases as it was extremely difficult to get consent from all patients. Additionally, we have only found one case of true carcinoma, and we are well aware that this number is quite low. However, of 104 cases which showed inflammation in liquid based cytology, 29 cases turned out to be CIN II or worse lesions with a false negative rate of $27.88 \%$. (Of those 29

\begin{tabular}{|l|c|c|c|}
\hline Project & TCT & HPV & DNA \\
\hline LBC cost & $30 \times 10^{4}$ & 0 & 0 \\
\hline HPV cost & 0 & $70 \times 10^{4}$ & 0 \\
\hline DNA cost & 0 & 0 & $30 \times 10^{4}$ \\
\hline $\begin{array}{l}\text { Number of positive } \\
\text { cases }\end{array}$ & 526 & 1685 & 483 \\
\hline Colposcopy cost & $526 \times 60$ & $1685 \times 60$ & $483 \times 60$ \\
\hline Biopsy cost & $263 \times 20$ & $842 \times 20$ & $241 \times 20$ \\
\hline Pathology cost & $263 \times 100$ & $842 \times 100$ & $241 \times 100$ \\
\hline CIN cases detected & 101 & 200 & 166 \\
\hline Total costs (RMB) & 363120 & 902140 & 357900 \\
\hline $\begin{array}{l}\text { Cost-effectiveness } \\
\text { ratio (cost/case) }\end{array}$ & 3595.25 & 4510.7 & 2156.02 \\
\hline
\end{tabular}

Table 8: Comparison of operating costs between the three screening methods.

cases 11 were CIN III. and 1 was cancer case). Conventional cytology results included 27 ASCUS cases, 4 LSIL and 0 HSIL cases, of which 10 cases were CIN II or worse lesions. Using ASCUS and worse lesions as the criteria for biopsy, the sensitivity of LBC was only $25.6 \%$ and negative predictive value was $72 \%$. Due to poor sensitivity and possible high rate of missed diagnoses, many patients with precancerous lesions or cervical cancer could have been overlooked if this was not a study. Normally they would not be followed up with biopsy assessment.

We tried to understand the reasons for low sensitivity in our data and why pathologists had such a difficult time in recognizing abnormal changes in the liquid based cytology slides (LBC). We came to a conclusion that there could be several possible factors. Again, there is a lack of specific cervical cytology screening training, which leads to overall poor performance. Furthermore, pathologists in primary care hospitals are usually overworked due to a huge load of various different tasks, such as grossing, histology reading, and many other daily services, which can lead to reading fatigue. Whatever is the underlying reason for low sensitivity in our study, it is obvious that if a cervical cancer screening would rely only on primary care hospital's pathologists and conventional cytology as the only test many pre cancer and cancers cases would be missed in much higher rate than suggested elsewhere [12]. Facing growing cervical cancer awareness and demand for testing, more standardized and preferably automated technology is surely one efficient option to address these burning issues, because it has been demonstrated that this technology should be used in order to improve quality in cervical cancer screening. Using automation would bring standardization across provinces' laboratories and would most likely improve overall performance in cervical cytology, particularly in China, where cytology professionals are relatively short-handed.

In view of the above problems, we looked at combined DNA ploidy as a possible tool, because automation and standardization could be readily implemented.

DNA nuclear changes have been reported earlier as a suitable method that can be used in cervical cytology for detecting cervical intraepithelial changes [13] when human cells are affected by 
carcinogenic agents, the immediate event begins at the chromosomal level and a change in their structure begins, leading to DNA content change and resulting in so called aneuploidy nuclei (cells).

In essence, cancer is a rapid and unlimited proliferation which leads towards uncontrolled cell multiplication and eventually metastasis. During this process nuclear DNA content changes and measuring those changes can be used as a direct reflection of tumor proliferation. Thus DNA content is an important biological indicator of cell malignant transformation [14]. Other authors also concluded that DNA changes which they call "Genomic Instability" [15] can be used as an important biomarker of carcinogenesis and progress in cervical cancer. Detection and measurement of those changes by quantifying DNA in nuclei allow automation of the whole process. This is particularly true today when computers are faster and can handle huge amount of data in no time. Those nuclear morphometric changes are used for classifying various objects captured on the slide. The entire process is automated, enhancing implementation of quality control procedure on every slide.

The present study showed that: of 31ASCUS and worse cases detected by conventional cytology, 10 were CIN II+. If LSIL was used for cut-off criteria for biopsy, conventional cytology (LBC) has a sensitivity of only $5 \%$ for CIN II+ cervical lesions. DNA ploidy assessment detects 85 cases with aneuploidy, of which 31 were CIN II+. DNA ploidy sensitivity and NPV of CIN II+ were $80 \%$ and $84 \%$, respectively, significantly better for DNA ploidy analysis than for conventional cytology.

A large number of studies have confirmed that over $99.7 \%$ of cervical cancer cases are caused by HPV infection [16]. Finding HPV in cervical sample indicates presence of HPV virus and identifies a highrisk group; however they do not immediately identify those women where cervical lesions already occur. The specificity of DNA ploidy analysis in our study was $44 \%$, higher than that of HPV testing which was only $25 \%$. We could not find a particular reason for such a low specificity. Other authors described too that care HPV has relatively good sensitivity with much lower specificity [17].

Our conclusion was that using combined DNA ploidy analysis to screen populations can reduce the number of women in high-risk groups by further narrowing down who would require immediate follow up. This method displays its value in cost-effective screening of cervical neoplasm, and avoids unnecessary waste of human and material resources (Table 8 ).

In our study, the specificity of DNA aneuploidy testing was lower than that of TBS but $44 \%$ higher than that of HPV. This is important to keep in mind especially if we are planning to implement screening for the large population. Implementing a test with higher specificity as well as good sensitivity can substantially reduce the number of women who need to see gynecologist for follow up testing and save the cost of the medical care. We also found that the cost of DNA testing although the same as conventional cytology (30RMB) has the best acceptable advantage over other testing costs when it comes to detecting one case of CIN (Table 8).

Our current focus was to screen as many women as possible, to find cervical lesions, treat them as early as possible with the goal to reduce the incidence of advanced cervical cancer, rather than screening a large number of women to find HPV infection and risk group only. It is most likely that combining two or even three tests could give better results than any one test alone; however we have not looked at the detailed possibility of such combinations, which would certainly elevate the overall cost.
Several published articles have suggested that DNA ploidy measurement equals liquid based cytology (LBC) and HPV testing combined in one test [18], thus we are confident that implementing automated quantitative DNA ploidy platform, which has both data DNA ploidy and morphology in one in healthcare facilities can greatly improve laboratory performance particularly in rural areas.

\section{Conclusion}

The present study of 3,300 cases using a standardized and automated cytopathology workflow platform potentially contributes to a new and detailed data of this technology. It is fairly to state that automated DNA ploidy image cytometry system which also contains morphology data is suitable for a large-scale screening program. Automated platform is objective in nature, fast and relatively accurate, immune to human error. This platform can improve the detection rate of precancerous lesions and cervical cancer; it does not require extensive training; it is easy to implement and allows clinicians' timely interventions when an early cervical lesion is detected. In a long term, this technology can reduce the incidence of cervical cancer in our population and reduce the healthcare expenses. Moreover, digitalization of the image can facilitate remote monitoring and cloud computing, which in turn can contribute to high quality control, and overcome the bottlenecks created by lack of trained pathologists.

\section{References}

1. Parkin DM (2006) The global health burden of infection-associated cancers in the year 2002. Int J Cancer 118: 3030-3044.

2. Zhao $F$ (2001) The status of cervical cancer and its precancerous lesions screening method. Chinese Journal of Medical Science 23: 638-641.

3. Zhang W, Li S, Li N (2006) 318 cases of cervical intraepithelial neoplasia clinical analysis. Journal of Clinical Oncology 11: 666-669.

4. Ministry of Health of the People's Republic of China. Chinese Health Statistical Yearbook. Beijing: Peking Union Medical College Press (2011) (In Chinese) Ministry of Health of the People 's Republic of China. China Health Statistics Yearbook. Beijing: China Union Medical University Press

5. Shi JF, Canfell K, Lew JB, Qiao YL (2012) The burden of cervical cancer in China: synthesis of the evidence. Int. J. Cancer 130: 641-652.

6. Nghiem VT, Davies KR, Beck JR, Follen M, Macaulay C, et al. (2015) Economic evaluation of DNA ploidy analysis vs liquid-based cytology for cervical screening. British Journal of Cancer 112: 1951-1957.

7. Passamonti B, Gustinucci D, Giorgi Rossi P, Cesarini E, Bulletti S, et al. (2017) Cervical human papilloma virus (HPV) DNA primary screening test: Results of a population-based screening programme in central Italy. J Med Screen 3: 153-162.

8. Lorenzato M, Caudroy S, Nou JM (2008) Contribution of DNA ploidy image cytometry to the management of ASC cervical lesions. Cancer 114: 263-269.

9. Sun X, Li Y, Che D, Yan X, Ling H, et al. (2005) Cervical cancer screening by using DNA quantitative cytology. Zhenduan Binglixue Zazhi 12: 12-16.

10. Lonky NM, Sadgeh M, Tsadik GW, Petitti D (1999) The clinical significance of the poor correlation of cervical dysplasia and cervical malignancy with referral cytologic results. Am J Obstet Gynecol 181: 560-566.

11. Wang Z (2009) Obstetrics and Gynecology. Beijing: People's Health Publishing House 531-532.

12. Castanon A, Landy R, Michalopoulos D, Bhudia R, Leaver $H$, et al. (2017) Systematic Review and Meta-Analysis of Individual Patient Data to Assess the Sensitivity of Cervical Cytology for Diagnosis of Cervical Cancer in Low- and Middle-Income Countries. J Glob Oncol 3: 524-538.

13. Sun XR, Wang J, Garner D, Palcic B (2005) Detection of cervical cance and high grade neoplastic lesion by a combination of liquid-based sampling preparation and DNA measurements using automated image cytometry. Cell Oncol 27: 33-41. 
Citation: Yan D, Jigeng B, Yuping Z, Jie SG, Yan Z, et al. (2017) Comparison of Three Screening Methods for Cervical Cancer in China - A Systematic Study of 3,300 Cases in Rural Areas of Shanxi Province. J Cytol Histol 8: 492. doi: 10.4172/2157-7099.1000492

Page 6 of 6

14. Böcking A (2015) Comparability of tumor-cytogenetics and -DNA-cytometry Molecular Cytogenetics 8: 28

15. Duarte CEM, Carvalho CR, da Silva-Filho AL (2014) Adaptation of image cytometry methodology for DNA ploidy analysis of cervical epithelium samples: A pilot study. Taiwanese Journal of Obstetrics \& Gynecology 53: 227-231.

16. Arbyn M, Castellsagué X, de Sanjosé S, Bruni L, Saraiya M, et al. (2011) Worldwide burden of cervical cancer in 2008. Ann Oncol 22: 2675-2686.
17. Obiri-Yeboah D, Adu-Sarkodje Y, Djigma F, Akakpo K, Aniakwa-Bonsu E, et al. (2017) Options in human papillomavirus (HPV) detection for cervical cancer screening: comparison between full genotyping and a rapid qualitative HPVDNA assay in Ghana. Gynecologic Oncology Research and Practice 4: 5.

18. Garner D (2014) Clinical application of DNA ploidy to cervical cancer screening: A review. World J Clin Oncol 10: 931-965. 